\title{
Primary pulmonary melanoma: a report of two cases
}

\author{
Mototsugu Watanabe ${ }^{1}$, Hiromasa Yamamoto ${ }^{1}$, Shinsuke Hashida ${ }^{1,2}$, Junichi Soh ${ }^{1,3}$, Seiichiro Sugimoto ${ }^{1}$, \\ Shinichi Toyooka ${ }^{1,2,3^{*}}$ and Shinichiro Miyoshi ${ }^{1}$
}

\begin{abstract}
Malignant melanoma is a refractory malignancy with a dismal prognosis. It generally arises from the skin in most cases, and cases of primary pulmonary malignant melanoma are rare and often behave aggressively. We have treated two cases of localized primary pulmonary malignant melanoma using surgical resection. Pulmonary malignant melanomas often metastasize to the brain and liver; one of our cases exhibited metastasis to the cecum at about 8 months after surgery. Because cutaneous melanomas often carry activating mutations in the BRAF gene (V600E), we performed a BRAF mutational analysis using direct sequencing for both of these tumors arising from the lung. However, no BRAF mutations were detected. We detected a p53 mutation, which was thought to be a potential somatic mutation, in one of the two cases using a sequencing panel targeting 20 lung cancer-related genes. Although we also checked the expression of programmed death ligand 1 (PD-L1) on the surface of the tumor cells by immunohistochemical testing, neither of our two cases expressed PD-L1. Further molecular analyses may uncover the characteristics of primary pulmonary malignant melanomas.
\end{abstract}

Keywords: Primary pulmonary malignant melanoma, Target sequencing, Surgical resection

\section{Background}

Malignant melanoma is an often fatal cutaneous neoplasm. As a primary tumor, malignant melanoma of the lung is rare, accounting for only $0.01 \%$ of all primary lung tumors [1]. To diagnose malignant melanoma as a primary tumor arising from the lung, the absence of primary melanoma lesions at cutaneous or nonpulmonary extracutaneous sites must be demonstrated [2].

Approximately 40 to $60 \%$ of cutaneous melanomas carry mutations in the BRAF gene (V600E), which leads to the constitutive activation of downstream signaling through the MAPK pathway [3, 4]. At present, whether pulmonary malignant melanomas carry $B R A F$ mutations has not been reported. Additionally, immunotherapy to inhibit programmed death 1 (PD-1)/programmed death ligand 1 (PD-L1) pathway that has a role for immune escape

\footnotetext{
* Correspondence: toyooka@md.okayama-u.ac.jp

'Department of Thoracic, Breast and Endocrinological Surgery, Okayama University Graduate School of Medicine, Dentistry and Pharmaceutical Sciences, Okayama 700-8558, Japan

${ }^{2}$ Department of Clinical Genomic Medicine, Okayama University Graduate School of Medicine, Dentistry and Pharmaceutical Sciences, 2-5-1 Shikata-cho, Kita-ku, Okayama 700-8558, Japan

Full list of author information is available at the end of the article
}

is developed for malignant melanoma [5, 6]. It is reported that the efficacy of anti-PD-1 antibody was related to the expression of PD-L1 in tumor cells [6]. Here, we describe two cases of possible primary pulmonary malignant melanoma with wild-type BRAF and no expression of PD-L1.

\section{Case presentation \\ Case 1}

A 66-year-old man with hemoptysis was referred to our hospital because of a pulmonary malignant tumor. A bronchoscopy revealed a tumor arising from the right middle lobe bronchus (Fig. 1a), and a histological diagnosis based on a bronchial biopsy revealed a malignant melanoma. A positron emission tomography-computed tomography (PET-CT) scan revealed no other lesions that could have represented a primary site (Fig. 1b). A right middle lobectomy with mediastinal and hilar lymphadenectomy was performed. Some tumor cells had melanin in the cytoplasm (Fig. 1c). Immunohistochemical analysis showed positive cytoplasmic staining of the tumor cells for HMB-45, Tyrosinase, and MART1, although the expression of TTF-1 in tumor cells was negative. The postoperative course was uneventful. Combination chemotherapy of 


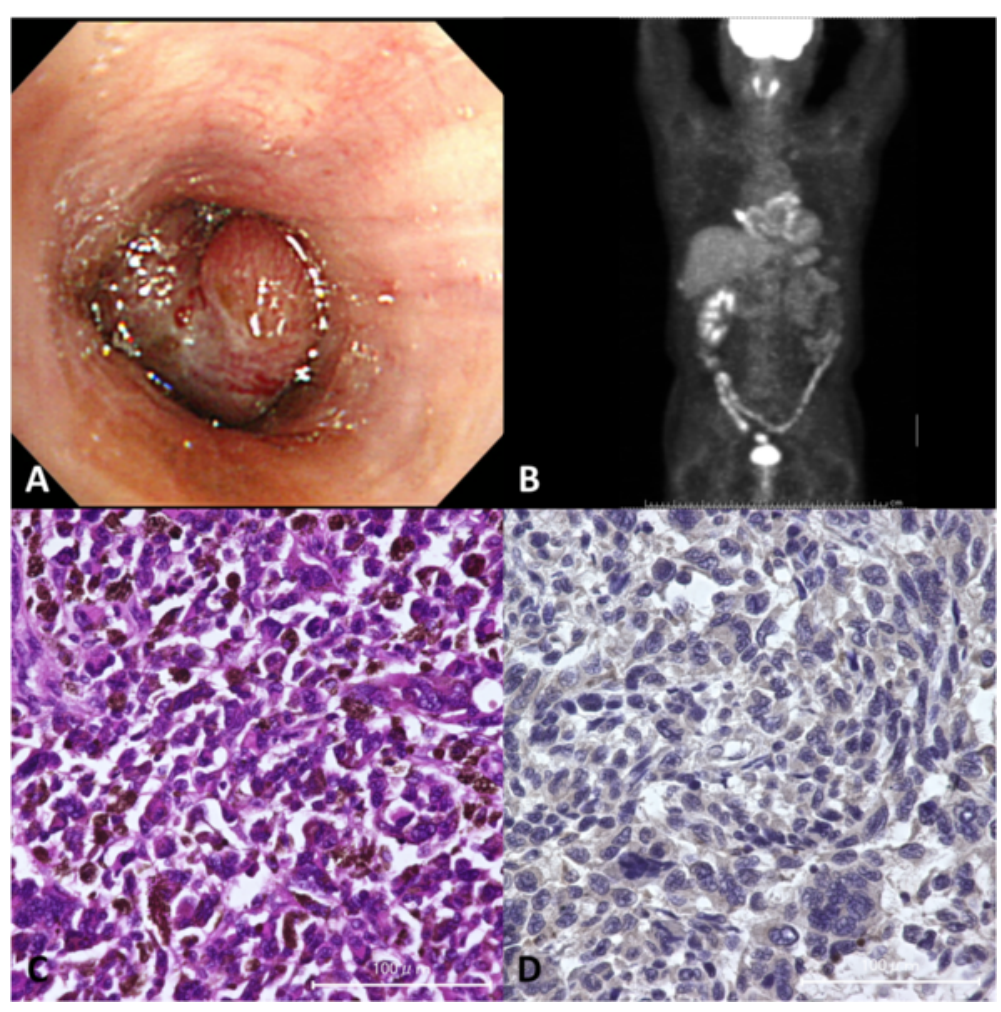

Fig. 1 Diagnostic images and pathological findings for case 1. a A bronchoscopy revealed a tumor arising from the right middle lobe bronchus. b PET-CT scan revealed no other lesions that could have represented a primary site. $\mathbf{c}$ Hematoxylin-eosin staining of the resected tumor, $\times 40$ (scale bar, $100 \mu \mathrm{m}$ ). d Immunohistochemistry showed negative for PD-L1 staining, $\times 40$ (scale bar, $100 \mu \mathrm{m}$ )

dacarbazine, nimustine, and vincristine was performed. At the time of the 22 months after surgery, he was alive without disease recurrence.

\section{Case 2}

A 46-year-old woman was found to have an abnormal shadow on a chest X-ray during an annual check-up. A CT and PET-CT scan showed a tumor in the left lower lobe of the lung (Fig. 2a, b). Pathological examination of a specimen obtained during a transbronchial lung biopsy revealed that the tumor was a malignant melanoma (Fig. 2c). Because further examinations did not reveal any other tumors that could have been the primary lesion, we diagnosed the lung tumor as a primary pulmonary malignant melanoma. A left lower lobectomy with mediastinal and hilar lymph node dissection was performed. Immunohistochemical staining was negative for S-100 protein, chromogranin, synaptophysin, keratin, and TTF-1 but positive for HMB-45 and vimentin. The postoperative course was uneventful. She refused to receive adjuvant chemotherapy. Eight months after the pulmonary resection, a PET-CT scan showed the uptake of fluorodeoxyglucose in the cecum (Fig. 2e). An ileocecal resection was performed, and the pathological examination indicated that the tumor was considered to be the recurrence of a melanoma.

\section{Molecular profiling}

We determined the $B R A F$ mutational status (exons 11 and 15) using direct sequencing in the two tumors. The detailed methods have been described previously [7]. However, we did not detect any BRAF mutations. Next, we performed a target sequencing analysis using the Human Lung Cancer Panel (Qiagen, Hilden, Germany), which targets 20 lung cancer-related genes including most of the exons in $B R A F$, using the same samples. Although various variants were detected using this analysis, only the p53 mutation (P72R) in case 1 was considered to be a potential somatic mutation after restricting the variants using GeneRead Software (Qiagen, Venlo, Netherlands) as shown in Table 1. P72R mutation has been already reported in bladder and gastric cancers, but there are no reports in lung cancer or malignant melanoma ([8], accessed on August 14, 2015).

Furthermore, we also checked the expression of PD-L1 on the surface of the tumor cells by immunohistochemical testing in formalin-fixed, paraffin-embedded tumor specimens with the use of a rabbit monoclonal antihuman PDL1 antibody. In the previous reports, PD-L1 positivity was defined as at least $5 \%$ of tumor cells showing cell-surface 


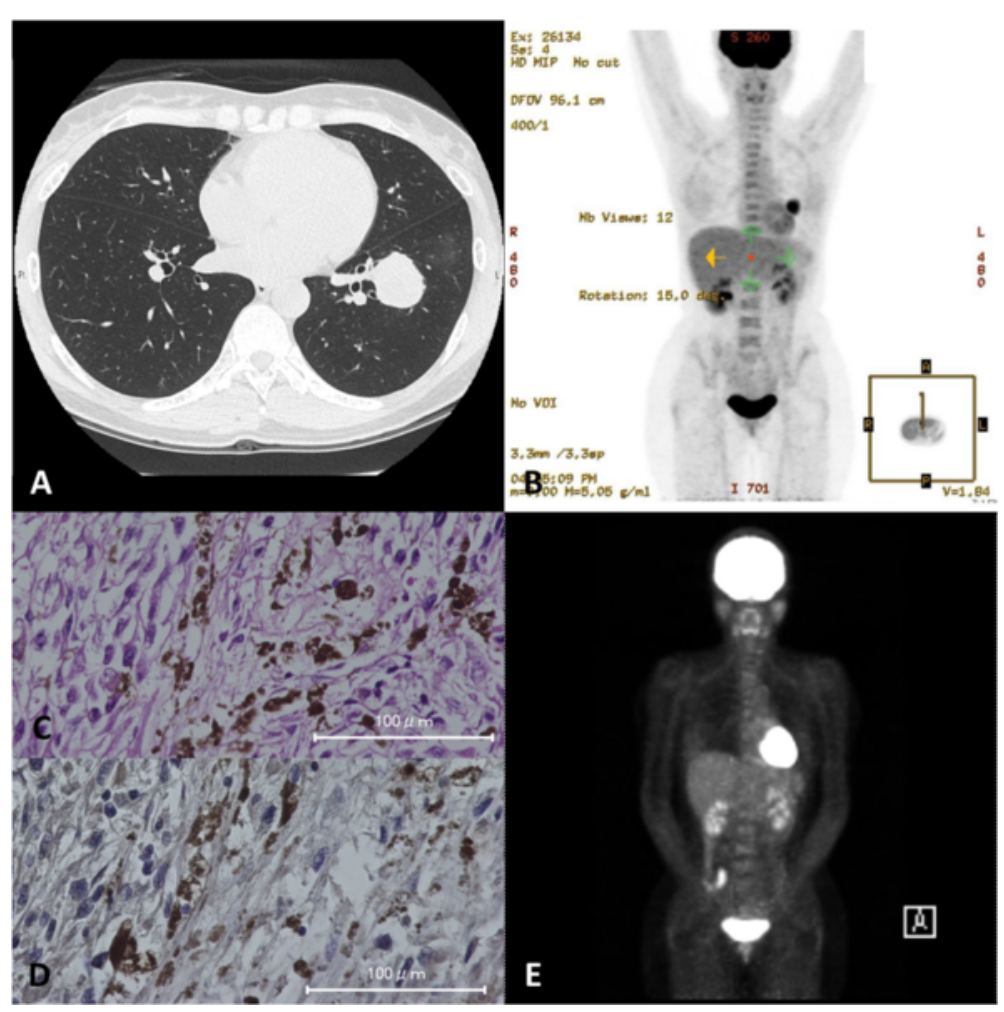

Fig. 2 Diagnostic images and pathological findings for case 2. a A CT scan shows a large tumor in the left lower lobe of the lung. b Preoperative PET-CT scan revealed no other lesions that could have represented a primary site. c Hematoxylin-eosin staining of the resected tumor, $\times 40$ (scale bar, $100 \mu$ m). $\mathbf{d}$ Immunohistochemistry showed negative for PD-L1 staining, $\times 40$ (scale bar, $100 \mu \mathrm{m})$. e Eight months after the pulmonary resection, PET-CT scan showed the uptake of fluorodeoxyglucose in the cecum

PD-L1 staining of any intensity in a section containing at least 100 tumor cells that could be evaluated $[9,10]$. According to the definition of PD-L1 positivity by them, neither of our two cases was considered to express PD-L1 (Figs. 1d and 2d).

\section{Discussion}

The total number of operations in general thoracic surgery is increasing year by year in Japan [11]. However, there are a few reports about primary pulmonary melanoma [2]. There are four clinical criteria that should be satisfied for the diagnosis of primary melanoma of the lung: (1) no previously removed skin tumor, unless the pathology examination did not show malignancy and the slides are still available for reevaluation; (2) no excised ocular tumor; (3) a solitary tumor in the surgical specimen from the lung; and (4) no demonstrable melanoma in other organs at the time of surgery $[1,12]$. Both of our cases fulfill the abovementioned diagnostic criteria.

Regarding treatment, surgery is necessary for a cure for localized pulmonary malignant melanoma because the effect of chemotherapy on malignant melanoma is limited [13]. Even among patients who undergo surgery, the prognosis of patients with malignant melanoma of the lung is generally poor because the lesion often metastasizes soon after surgery. Regarding metastatic spread, primary pulmonary melanomas often metastasize to the brain and the liver [2, 14-16], similar to other types of primary lung cancer. Intriguingly, one of our cases exhibited metastasis to the cecum. To the best of our knowledge, this is the first report describing a malignant melanoma of the lung metastasizing to the cecum.

$B R A F$-activating mutation is a characteristic of malignant melanoma, and molecular-targeted therapy for BRAF has been developed [17]. However, our two cases did not exhibit activating mutations in $B R A F$. Considering the fact that the presence of $B R A F$-activating mutations has not been reported in pulmonary malignant melanoma, their features may differ from those of primary skin melanoma. A target sequencing analysis did not detect any possible somatic driver mutations commonly observed in lung cancer [18], such as EGFR, HER2, KRAS, ALK, MET, or $P I K 3 C A$, suggesting that primary pulmonary malignant melanomas may have different oncogenic pathways from primary lung cancer from the viewpoint of driver oncogenes.

In these days, there are many reports about the efficacy of anti-PD-1 antibody for cutaneous melanoma patients 
Table 1 Mutational status of 20 genes in cases 1 and 2

\begin{tabular}{|c|c|c|}
\hline Gene name & Case 1 & Case 2 \\
\hline MTOR & WT & WT \\
\hline NRAS & WT & WT \\
\hline PTGS2 & WT & WT \\
\hline$A L K$ & WT & WT \\
\hline CTNNB1 & WT & WT \\
\hline PIK3CA & WT & WT \\
\hline PDGFRA & WT & WT \\
\hline KIT & WT & WT \\
\hline EGFR & WT & WT \\
\hline MET & WT & WT \\
\hline BRAF & WT & WT \\
\hline CDKN2A & WT & WT \\
\hline PTEN & WT & WT \\
\hline HRAS & WT & WT \\
\hline KRAS & WT & WT \\
\hline$R B 1$ & WT & WT \\
\hline AKT1 & WT & WT \\
\hline TP53 & P72R & WT \\
\hline ERBB2 & WT & WT \\
\hline STK11 & WT & WT \\
\hline
\end{tabular}

WT wild type

and a potential association between the occurrence of a response and expression of $\mathrm{PD}-\mathrm{L} 1$ in patients receiving nivolumab [10]. On the other hand, Robert et al. reported that regardless of PD-L1 status, nivolumab-treated patients had improved overall survival, as compared with dacarbazine-treated patients [19]. Although our findings did not show the positivity of PD-L1 in the two cases, there is still the possibility of the efficacy of immunotherapy for primary pulmonary melanoma, as the role of PD-1/PD-L1 pathway in primary pulmonary melanoma is yet to be investigated.

\section{Conclusions}

In conclusion, we encountered two cases of malignant melanoma of the lung that did not carry activating mutations in the $B R A F$ gene. Further molecular analyses may uncover the characteristics of primary malignant melanoma.

\section{Consent}

Written informed consent was obtained from the patients for publication of this case report and any accompanying images. A copy of the written consent is available for review by the editor in chief of this journal.

\section{Abbreviations}

PD-1: Programmed death 1; PD-L1: Programmed death ligand 1;

PET-CT: Positron emission tomography-computed tomography.

\section{Competing interests}

The authors declare that they have no competing interests.

\section{Authors' contributions}

MW, HY, ST, and SM conceived the project. MW and SH performed the experiments. MW, HY, JS, SS, and ST collected the data and assisted with the experiments. MW, HY, and SH analyzed the data. MW, HY, SH, JS, and ST prepared the manuscript with input from the other authors. ST and SM supervised the project. All authors read and approved the final manuscript.

\section{Acknowledgements}

We thank Dr. Takehiro Matsubara (Biobank of Okayama University Hospital, Okayama, Japan) for his technical support and Dr. Noriyuki Tanaka (Department of Pathology, Okayama University Hospital) for providing advice.

\section{Author details}

'Department of Thoracic, Breast and Endocrinological Surgery, Okayama University Graduate School of Medicine, Dentistry and Pharmaceutical Sciences, Okayama 700-8558, Japan. ²Department of Clinical Genomic Medicine, Okayama University Graduate School of Medicine, Dentistry and Pharmaceutical Sciences, 2-5-1 Shikata-cho, Kita-ku, Okayama 700-8558, Japan. ${ }^{3}$ Biobank of Okayama University Hospital, Okayama 700-8558, Japan.

Received: 27 April 2015 Accepted: 7 September 2015

Published online: 17 September 2015

\section{References}

1. Wilson RW, Moran CA. Primary melanoma of the lung: a clinicopathologic and immunohistochemical study of eight cases. Am J Surg Pathol. 1997;21:1196-202.

2. Maeda R, Isowa N, Onuma H, Miura H, Tokuyasu H, Kawasaki Y. Primary malignant melanoma of the lung with rapid progression. Gen Thorac Cardiovasc Surg. 2009;57:671-4.

3. Davies H, Bignell GR, Cox C, Stephens P, Edkins S, Clegg S, et al. Mutations of the BRAF gene in human cancer. Nature. 2002;417:949-54.

4. Menzies AMLT, Wilmott JS, Hyman J, Kefford RF, Thompson JF, OToole S, et al. Intrapatient homogeneity of BRAF ${ }^{\mathrm{V} 600 \mathrm{E}}$ expression in melanoma. Am J Surg Pathol. 2014;38:377-82.

5. Schreiber RD, Old LJ, Smyth MJ. Cancer immunoediting: integrating immunity's roles in cancer suppression and promotion. Science. 2011;331:1565-70.

6. Topalian SL, Hodi FS, Brahmer JR, Gettinger SN, Smith DC, McDermott DF, et al. Safety, activity, and immune correlates of anti-PD-1 antibody in cancer. N Engl J Med. 2012;366:2443-54.

7. Yamamoto H, Shigematsu H, Nomura M, Lockwood WW, Sato M, Okumura N, et al. PIK3CA mutations and copy number gains in human lung cancers. Cancer Res. 2008;68:6913-21.

8. cBioPortal for Cancer Genomics. [http://www.cbioportal.org/].

9. Taube JM, Anders RA, Young GD, Xu H, Sharma R, McMiller TL, et al. Colocalization of inflammatory response with $\mathrm{B} 7-\mathrm{H} 1$ expression in human melanocytic lesions supports an adaptive resistance mechanism of immune escape. Sci Transl Med. 2012:4:27-37.

10. Wolchok JD, Kluger H, Callahan MK, Postow MA, Rizvi NA, Lesokhin AM, et al. Nivolumab plus ipilimumab in advanced melanoma. N Engl J Med. 2013;369:122-33.

11. Committee for Scientific Affairs TJAfTS, Masuda M, Kuwano H, Okumura M, Amano J, Arai H, et al. Thoracic and cardiovascular surgery in Japan during 2012 : annual report by the Japanese association for thoracic surgery. Gen Thorac Cardiovasc Surg. 2014;62:734-64.

12. Jensen OAEJ. Primary malignant melanoma. Scand J Respir Dis. 1967;48:127-35.

13. Bajetta E, Del Vecchio M, Bernard-Marty C, Vitali M, Buzzoni R, Rixe O, et al. Metastatic melanoma: chemotherapy. Semin Oncol. 2002;29:427-45.

14. Neri S, Komatsu T, Kitamura J, Otsuka K, Katakami N, Takahashi Y. Malignant melanoma of the lung: report of two cases. Ann Thorac Cardiovasc Surg. 2011;17:170-3.

15. Gong L, Liu X-Y, Zhang W-D, Zhu S-J, Yao L, Han X-J, et al. Primary pulmonary malignant melanoma. A clinicopathologic study of two cases. Diagn Pathol. 2012;7:123. 
16. Ouarssani A, Atoini F, Reda R, Lhou FA, Rguibi MI. Malignant melanoma of the lung: a case report. Pan Afr Med J. 2012;11:68.

17. Chapman PB, Hauschild A, Robert C, Haanen JB, Ascierto P, Larkin J, et al. Improved survival with vemurafenib in melanoma with BRAF V600E mutation. N Engl J Med. 2011;364:2507-16.

18. Toyooka S, Mitsudomi T, Soh J, Aokage K, Yamane M, Oto T, et al. Molecular oncology of lung cancer. Gen Thorac Cardiovasc Surg. 2011;59:527-37.

19. Robert C, Long GV, Brady B, Dutriaux C, Maio M, Mortier L, et al. Nivolumab in previously untreated melanoma without BRAF mutation. N Engl J Med. 2015;372:320-30.

Submit your next manuscript to BioMed Central and take full advantage of:

- Convenient online submission

- Thorough peer review

- No space constraints or color figure charges

- Immediate publication on acceptance

- Inclusion in PubMed, CAS, Scopus and Google Scholar

- Research which is freely available for redistribution 